\title{
A Renormalization Prescription for Massless Quantum Electrodynamics
}

\author{
T. R. Hurd * \\ Department of Mathematics, University of British Columbia, Vancouver, British Columbia, \\ V6T 1Y4, Canada
}

\begin{abstract}
A new perturbative renormalization scheme is introduced for massless $\mathrm{QED}_{4}$. The method rests on the renormalized tree expansion rather than dimensional renormalization, and so does not rely on any detailed analyticity arguments. The infrared convergence of the gauge invariant prescription is seen to be a consequence of the Ward identity satisfied by the theory.
\end{abstract}

\section{Introduction}

Perturbative $\mathrm{QED}_{4}$ with a massless electron requires a renormalization scheme which eliminates ultraviolet (UV) and infrared (IR) divergences while preserving a Ward identity such as that satisfied formally by the unrenormalized theory

$$
Z=\int d A d \tilde{\psi} d \psi \exp -\int\left[\frac{1}{4} F^{2}-\frac{1}{2}(\partial \cdot A)^{2}+\tilde{\psi}(-i \not \partial+m+|e| A) \psi\right] .
$$

The general method of dimensional renormalization pioneered by 't Hooft and Veltman ['tHV] and refined by Becchi, Rouet, and Stora [BRS] and Breitenlohner and Maison [BM] apparently provides us with just such a scheme. Here, we present a direct alternative proof of the perturbative gauge invariance and renormalizability of massless $\mathrm{QED}_{4}$ (using the Pauli-Villars loop/photon regularization). It is based on the tree expansion renormalization method introduced by Gallavotti and Nicolo [GN], as developed and applied to massive QED $_{4}$ by Feldman, Hurd, Rosen, and Wright [FHRW]. The proof of the $m \rightarrow 0$ limit, not considered in [FHRW], follows the lines anticipated in [BM]: "A proof of the Ward identities expressing the symmetry at the level of Green's (or vertex) functions can be given to all orders of perturbation theory inductively hand in hand with a proof of the absence of IR-counterterms in the Lagrangian."

\footnotetext{
^ Research supported by the Natural Sciences and Engineering Research Council
} 
The Feynman graph

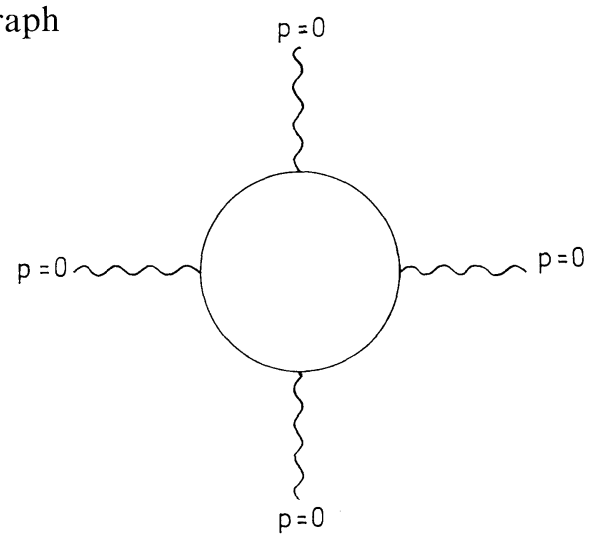

illustrates the general point: in the presence of a fixed UV cutoff $A$, this graph is finite as the electron mass $m_{e} \rightarrow 0$, although logarithmically divergent by power counting. This fact follows directly from the identity

$$
\operatorname{Tr}\left[2 k \gamma^{\mu} k \gamma_{\mu} k \gamma^{v} k \gamma_{v}+k \gamma^{\mu} k \gamma^{v} k \gamma_{\mu} k \gamma_{v}\right]=0 \text { for all } k \in R^{4} \text {. }
$$

This type of cancellation of logarithmic IR divergence occurs to all orders in perturbation theory.

Why might the present method be of interest? Firstly, dimensional regularization requires analyticity arguments which are disturbingly abstract, and for QED can be replaced by the simpler Pauli-Villars regularization. Secondly, the tree expansion method of [GN] and [FHRW] constitutes a very general and very concrete proof of UV and IR regular bounds on perturbation theory. The treatment of $\mathrm{QED}_{4}$ with $m_{e}>0$ shows how abelian gauge invariance can also be handled, but the limiting $m_{e}=0$ case is not an immediate consequence of the method of [FHRW]. Thirdly, the present method is an illustration of how Ward identities can be used to control quantities which are IR divergent by power counting, a fact which will be of interest in non-abelian models. Fourthly, the renormalization conditions of dimensional renormalization are based on "minimal subtraction" while the IR method of [FHRW] imposes BPHZ-type renormalization conditions. The explicit relation between the perturbation expansions defined by these two methods is not clear. Finally, the simplicity and naturalness of the massless QED Lagrangian make a simple and natural renormalization method desirable.

In outline, Sect. 2 consists of a summary of relevant facts about massive QED $_{4}$ proved in [FHRW], leading to a statement of the main convergence result for massless $\mathrm{QED}_{4}$ (Theorem 2.1). The proof of this result contained in Sect. 3 requires a Ward identity (Lemma 3.2) whose proof is given in Sect. 4. The method presented here rests heavily on the notation and results of [FHRW]. The reader is urged to consult this reference for proofs of some of the facts stated here. 


\section{A Renormalization Prescription for Massless QED $_{4}$}

Massless euclidean quantum electrodynamics consists of a vector field $A_{\mu}$ and spinor fields $\tilde{\psi}, \psi$ and lagrangian density

$$
\mathscr{L}=\frac{1}{4} F_{\mu \nu} F^{\mu \nu}+\frac{1}{2}\left(\partial_{\mu} A^{\mu}\right)^{2}+\tilde{\psi}(-i \phi) \psi+e \tilde{\psi} \not \psi \psi
$$

Here

$$
\not A=\gamma^{\mu} A_{\mu}, \quad \phi=\gamma^{\mu} \partial_{\mu},
$$

where the $4 \times 4$ antihermitian euclidean Dirac matrices satisfy

$$
\gamma^{\mu} \gamma^{v}+\gamma^{v} \gamma^{\mu}=-2 \delta^{\mu \nu},
$$

and $F_{\mu \nu}=\partial_{\mu} A_{v}-\partial_{v} A_{\mu}$ and $e$ is the bare electric charge. The free field photon covariance is $D^{\mu v}(x, y)=\delta^{\mu v}\left(-\partial^{2}\right)_{x y}^{-1}$ and the free field electron covariance $S(x, y)$ $=(i \phi)_{x y}^{-1}$.

We consider the gauge invariant IR regularization of massless QED obtained by giving the free photon and electron propagators a small mass $M^{I},-\infty<I<0$, $M>1$ :

$$
D_{I}^{\mu \nu}(x, y)=\delta^{\mu \nu}\left(-\partial^{2}+M^{2 I}\right)_{x y}^{-1} ; \quad S_{I}(x, y)=\left(-i \phi+M^{I}\right)_{x y}^{-1} .
$$

We choose a smooth scale decomposition

$$
D_{I}=\sum_{k=I}^{\infty} D_{I}^{(k)} ; \quad S_{I}=\sum_{k=I}^{\infty} S_{I}^{(k)},
$$

subject to bounds [FHRW, Eq. (1.15)] which imply that $D_{\bar{I}}^{\geq k}$ and $S_{\bar{I}}^{\geq k}$ are exponentially decaying on the length scale $M^{-2 k}$, that $D_{I}^{<k}\left(S_{I}^{<k}\right)$ is bounded by $O(1) M^{2 k}\left(O(1) M^{3 k}\right)$ at short distance, and further such conditions.

The renormalized perturbation theory consists of the effective potentials $\left\{V_{r}^{I}(\Phi) ; r=e, I, I+1, \ldots\right\}, \Phi \equiv(A, \tilde{\psi}, \psi)$. In particular, the external effective potential $V_{e}^{I}$ is the generating functional for connected, amputated, euclidean Green's functions. Each $V_{r}^{I}$ is defined by its renormalized tree expansion, which is completely determined by (2.1), (2.2), and a set of renormalization conditions. Renormalization conditions are specified in terms of the localization operators $L_{r}$, which are specific projection operators from functions of $\Phi$ onto the span of the following local functions (of nonnegative scaling dimension):

$$
\begin{aligned}
& V_{1}=\int: \tilde{\psi} \psi:, \quad V_{2}=\int: A_{\mu} A^{\mu}: \quad \text { (positive dimension), } \\
& V_{3}=\int: F_{\mu v} F^{\mu v}:, \quad V_{4}=\int: \tilde{\psi}(-i \oint) \psi:, \quad V_{5}=\int: \tilde{\psi} \not t \psi: \quad \text { (marginal and } \\
& \text { gauge invariant), (2.3) } \\
& V_{6}=\int:\left(A_{\mu} A^{\mu}\right)^{2}:, \quad V_{7}=\int:\left(\partial_{\mu} A^{\mu}\right)^{2}: \quad \text { (marginal and non } \\
& \text { gauge invariant). }
\end{aligned}
$$

We write $L=L^{+}+L^{0, \text { inv }}+L^{0, \text { ninv }}$.

The renormalization conditions

$$
\left.\begin{array}{rl}
L V_{e}^{I} & =-e V_{5} \\
\lim _{r \rightarrow \infty}(1-L) V_{r}^{I} & =0,
\end{array}\right\}
$$


lead to a consistent UV renormalization, by [FRHW, Theorem 2.6] [the notation " $\lim _{r \rightarrow \infty}(1-L) V_{r}^{I}=0$ " is used as short-hand for renormalization schemes achieved from the $N \rightarrow \infty$ limit of UV regularized effective potentials $V_{r}^{I, N}$ satisfying $\left.(1-L) V_{N}^{I, N}=0\right]$. Also, since (2.4) gives $L^{0, \text { ninv }} V_{e}^{I}=0$, the argument which leads to [FHRW, Theorem 5.3] works, and shows that the resulting perturbation theory is of gauge invariant type with physical charge given by the parameter $e$.

Conditions (2.4) lead to a divergent $I \rightarrow-\infty$ limit. In general, when marginal terms in the external effective potential are specified, elementary power counting indicates a divergent limit. By the general IR analysis of [FHRW, Sect. 6], renormalization conditions which specify marginal terms of an intermediate effective potential, such as

$$
\left.\begin{array}{rl}
L^{+} V_{e}^{I} & =0 \\
L^{0} V_{0}^{I} & =-e V_{5} \\
\lim _{r \rightarrow \infty}(1-L) V_{r}^{I} & =0
\end{array}\right\}
$$

do yield an IR limit. But such prescriptions are dependent on the form of the scale decomposition (2.2), and in particular will not usually yield a theory with the required gauge invariance.

We adopt the renormalization condition

$$
\left.\begin{array}{rl}
\left(L^{+}+L^{0, \mathrm{ninv}}\right) V_{e}^{I} & =0 \\
L^{0 . \mathrm{inv}} V_{0}^{I} & =-e V_{5} \\
\lim _{r \rightarrow \infty}(1-L) V_{r}^{I} & =0
\end{array}\right\}
$$

and claim that it yields a gauge invariant theory with a well-defined IR limit. Since $L^{0, \text { inv }} V_{e}^{I}=0$ for (2.6) as for (2.4), gauge invariance can be expected. What is not so obvious is the existence of the $I \rightarrow-\infty$ limit, since (2.6) specifies marginal terms of $V_{e}^{I}$.

We can write

$$
L V_{e}^{I}=L^{0, \text { inv }} V_{e}^{I}=-a(I) V_{4}-e c(I) V_{5}-d(I) V_{3},
$$

where the coefficients $a, c, d$ are certain formal power series (fps) in the parameter $e$,

$$
a(I)=\sum_{n=2}^{\infty} a_{n}(I) e^{n}, \quad c(I)=1+\sum_{n=2}^{\infty} c_{n}(I) e^{n}, \quad d(I)=\sum_{n=2}^{\infty} d_{n}(I) e^{n},
$$

calculable via the renormalized tree expansion.

The techniques of [FHRW, Sects. 3-5] make use of the gauge invariant form of $L V_{e}^{I}$ to show that $V_{e}^{I}$ satisfies the Ward identity

$$
\begin{aligned}
V_{e}^{I}(A, \tilde{\psi}, \psi)= & V_{e}^{I}\left(A+\varepsilon(I) \partial \chi, \tilde{\psi} e^{i e \chi}\left(1+X_{0} S_{I}\right),\left(1+S_{I} X_{0}\right) e^{-i e \chi} \psi\right) \\
& +\int \tilde{\psi} e^{i e \chi}\left(X_{0}+X_{0} S_{I} X_{0}\right) e^{-i e \chi} \psi
\end{aligned}
$$

for any function $\chi(x)$, where $X_{0} \equiv e \not \chi$ and $\varepsilon(I) \equiv c(I)^{-1}(1+a(I))$. This means that for each $I$ the theory is gauge invariant with gauge parameter

$$
\tilde{e}(I)=e \varepsilon(I)^{-1} \text {. }
$$


We can also write

$$
L^{0} V_{0}^{I}=-e V_{5}-f(I) V_{7}-g(I) V_{6},
$$

where $f(I)=\sum_{n=2, \infty} f_{n}(I) e^{n}$ and $g(I)=\sum_{n=4, \infty} g_{n}(I) e^{n}$. Elementary IR power counting indicates that both $f$ and $g$ are logarithmically divergent as $I \rightarrow-\infty$. This observation notwithstanding, we shall see in Sect. 3 that $f \equiv \lim _{I \rightarrow-\infty} f(I)$ and $g \equiv \lim _{I \rightarrow-\infty} g(I)$ do exist, and since (2.6) is equivalent to

$$
\left.\begin{array}{rl}
L^{+} V_{e}^{I} & =0 \\
L^{0} V_{0}^{I} & =-e V_{5}-f(I) V_{6}-g(I) V_{7} \\
\lim _{r \rightarrow \infty}(1-L) V_{r}^{I} & =0,
\end{array}\right\}
$$

the existence of these limits is necessary and sufficient for the IR convergence of the theory (by [FHRW, Theorem 6.7]).

Theorem 2.1. For fixed $I,-\infty<I<0$, let $\left\{V_{r}^{I}\right\}$ be the family of effective potentials for the theory defined by the renormalization conditions (2.6). Then

$$
V_{r}=\lim _{I \rightarrow-\infty} V_{r}^{I} \text { and } V_{e}=\lim _{r \rightarrow-\infty} V_{r}
$$

exist in perturbation theory. Moreover

$$
\tilde{e}=\lim _{I \rightarrow-\infty} \tilde{e}(I)
$$

exists, and $V_{e}$ satisfies the Ward identity with this gauge parameter:

$$
\begin{aligned}
V_{e}(A, \tilde{\psi}, \psi)= & V_{e}\left(A+\partial \chi, \tilde{\psi} e^{i \tilde{e} \chi}(1+X S),(1+S X) e^{-i \tilde{e} \chi} \psi\right) \\
& +\int \tilde{\psi} e^{i \tilde{e} \chi}(X+X S X) e^{-i \tilde{e} \chi} \psi,
\end{aligned}
$$

for all Schwartz functions $\chi$, where $X \equiv \tilde{e} \phi \chi$.

\section{Proof of IR Convergence}

The proof of Theorem 2.1 is inductive on the order of perturbation theory. We assume that

$$
\varepsilon_{j-1}=\lim _{I \rightarrow-\infty} \varepsilon_{j-1}(I), \quad f_{j}=\lim _{I \rightarrow-\infty} f_{j}(I), \quad g_{j}=\lim _{I \rightarrow-\infty} g_{j}(I)
$$

exist for each $2 \leqq j \leqq n$ and use a Ward identity for $V_{0}^{I}$ to deduce the existence of the $\operatorname{limits} \varepsilon_{n}, f_{n+1}, g_{n+1}$. Note that $\tilde{e}$ exists to order $e^{n}$ if and only if $\varepsilon$ exists to order $e^{n-1}$.

The propagators $D_{I}$ and $S_{I}$ are decomposed into hard and soft terms:

$$
\begin{aligned}
& D_{I}=\sum_{k=I}^{0} D_{I}^{(k)}+\sum_{k=1}^{\infty} D_{I}^{(k)} \equiv D_{I}^{s}+D_{I}^{h}, \\
& S_{I}=\sum_{k=I}^{0} S_{I}^{(k)}+\sum_{k=1}^{\infty} S_{I}^{(k)} \equiv S_{I}^{s}+S_{I}^{h} .
\end{aligned}
$$


We suppose that $S^{s}$ can be obtained from $S$ by convolution against a smooth exponentially decaying monotone function $\eta$ :

$$
S_{I}^{s}(x)=(2 \pi)^{-2} \int \eta(x, y) S_{I}(y) d y
$$

[take for example $\left.\eta(x, y)=(2 \pi)^{-2} e^{-(x-y)^{2} / 2}\right]$. The hard propagators are exponentially damped on the scale 1 while the soft propagators are smooth and $O(1)$ at short distances. Recall the relation between $V_{0}^{I}$ and $V_{e}^{I}$ :

$$
V_{e}^{I}\left(\Phi^{e}\right)=\log \int d P^{s}(\Phi) \exp V_{0}^{I}\left(\Phi+\Phi^{e}\right)
$$

where $d P^{s}(\Phi)$ is the free field (gaussian) functional "measure" with covariance $D_{I}^{s} \oplus S_{I}^{s}$.

Let us express $V_{0}^{I}$ as a sum over Wick monomials

$$
\begin{aligned}
V_{0}^{I}= & \sum_{p, q \geqq 0} \int d \vec{x} d \vec{y} d \vec{z} V_{p q}^{I}(\vec{x} ; \vec{y} ; \vec{z}) \\
& \times: \tilde{\psi}\left(x_{1}\right) \ldots \tilde{\psi}\left(x_{q}\right) A\left(y_{1}\right) \ldots A\left(y_{p}\right) \psi\left(z_{1} \ldots\right) \psi\left(z_{q}\right):
\end{aligned}
$$

(this is a finite sum order by order in perturbation theory). Since, under the inductive hypothesis (3.1), the renormalization conditions (2.12) are finite as $I \rightarrow-\infty$, up to order $e^{n}$, it follows that the kernels up to order $e^{n}, V_{(\leqq n) p q}^{I}$, obey the standard I-uniform power counting bounds of [FHRW, Theorem 6.7]. In particular,

Lemma 3.1. If the limits (3.1) exist for $2 \leqq j \leqq n$, then the limits $V_{(\leqq n) p q}^{-\infty}(\vec{x})$ exist as distributions in the difference variables $x_{i}-x_{1}$ integrable against polynomially bounded smooth test functions.

The following identity is proved in Sect. 4.

Lemma 3.2. For any polynomially bounded smooth function $\chi$ :

$$
\begin{aligned}
0= & \varepsilon(I) \int\left[\partial_{A} V\right]^{\mu}\left(\Phi^{e}, x\right)\left[\partial_{\mu} \chi\right](x) d x \\
& +e \int \tilde{\psi}^{e}(x)[\phi \chi](x) \psi^{e}(x) d x \\
& +i e \int:\left[\partial_{\psi} V\right]\left(\Phi^{e}, x\right)\left[\eta(x, y)+M^{I} S_{I}^{h}(x, y)\right] \chi(y) \psi^{e}(y): d x d y \\
& +i e \int: \tilde{\psi}^{e}(x) \chi(x)\left[\eta(x, y)+M^{I} S_{I}^{h}(x, y)\right]\left[\partial_{\tilde{\psi}} V\right]\left(\Phi^{e}, y\right): d x d y \\
& -i e \int \operatorname{Tr}\left\{\left[\partial_{\tilde{\psi} \psi}^{2} V\right]\left(\Phi^{e}, x, y\right) \chi(z)\right. \\
& \times\left[\left(S_{I}^{s}+S_{I}^{h}\right)(y, z) \eta(z, x)-\eta(y, z)\left(S_{I}^{s}+S_{I}^{h}\right)(z, x)\right. \\
& \left.\left.+M^{I} S_{I}^{s}(y, z) S_{I}^{h}(z, x)+M^{I} S_{I}^{h}(y, z) S_{I}^{s}(z, x)\right]\right\} d x d y d z \\
& -i e \int \operatorname{Tr}\left\{\left[\partial_{\tilde{\psi}} V\right]\left(\Phi^{e}, x\right)\left[\partial_{\psi} V\right]\left(\Phi^{e}, y\right)\right. \\
& \left.\times\left(S_{I}^{h}(y, z) \eta(z, x)-\eta(y, z) S_{I}^{h}(z, x)\right)\right\} \chi(z) d x d y d z .
\end{aligned}
$$

Here

$$
\begin{gathered}
{\left[\partial_{\psi} V\right]\left(\Phi^{e}, x\right) \equiv \frac{\overleftarrow{\delta} V_{0}^{I}\left(\Phi^{e}\right)}{\delta \psi(x)}, \quad\left[\partial_{\tilde{\psi}} V\right]\left(\Phi^{e}, x\right) \equiv \frac{\vec{\delta} V_{0}^{I}\left(\Phi^{e}\right)}{\delta \tilde{\psi}(x)},} \\
{\left[\partial_{\tilde{\psi} \psi}^{2} V\right]\left(\Phi^{e}, x, y\right) \equiv \frac{\grave{\delta} \overleftarrow{\delta} V_{0}^{I}\left(\Phi^{e}\right)}{\delta \tilde{\psi}(x) \delta \psi(y)}, \quad\left[\partial_{A} V\right]^{\mu}\left(\Phi^{e}, x\right) \equiv \frac{\delta V_{0}^{I}\left(\Phi^{e}\right)}{\delta A_{\mu}(x)} .}
\end{gathered}
$$


We extract the dimension zero part of identity (3.5), where for power counting purposes the field $\chi$ is taken with scaling dimension zero. The only monomials in $(A, \tilde{\psi}, \psi, \chi)$ which arise are

$$
\begin{aligned}
& \mathscr{L}_{1} \equiv \int: \tilde{\psi}(x)[\phi \chi](x) \psi(x): d x, \\
& \mathscr{L}_{2} \equiv \int:\left[\partial_{\mu} A^{\mu}\right](x)\left[\partial_{\mu} \partial^{\mu} \chi\right](x): d x, \\
& \mathscr{L}_{3} \equiv \int:\left[A_{\mu} A^{\mu}\right](x)\left[A_{\mu} \partial^{\mu} \chi\right](x): d x
\end{aligned}
$$

[other possible monomials drop out by Furry's theorem and the fact that (3.5) depends only on $\left.\partial_{\mu} \chi\right]$.

The first term $T^{1}$ of (3.5) yields the objects of interest:

$$
L^{0} T^{1}=\varepsilon(I)\left[-e \mathscr{L}_{1}-2 f(I) \mathscr{L}_{2}-4 g(I) \mathscr{L}_{3}\right] .
$$

The second term is

$$
L^{0} T^{2}=e \mathscr{L}_{1}
$$

The last four terms of (3.5) require more manipulation. Let us write:

$$
\begin{aligned}
T^{i}= & e \sum_{p, q \geqq 0} \int d \vec{x} d \vec{y} d \vec{z} d w W_{p q}^{i}(\vec{x} ; \vec{y} ; \vec{z} ; w) \\
& \times: \tilde{\psi}\left(x_{1}\right) \ldots \tilde{\psi}\left(x_{q}\right) A\left(y_{1}\right) \ldots A\left(y_{p}\right) \psi\left(z_{1}\right) \ldots \psi\left(z_{q}\right) \chi(w):, \quad i=3,4,5,6 .
\end{aligned}
$$

Then

$$
L^{0} T^{i}=e \alpha_{1}^{i}(I) \mathscr{L}_{1}+e \alpha_{2}^{i}(I) \mathscr{L}_{2}+e \alpha_{3}^{i}(I) \mathscr{L}_{3}, \quad i=3,4,5,6,
$$

with coefficients given by the integrals

$$
\begin{aligned}
\alpha_{1}^{i}(I) \gamma^{\mu} & =\int W_{01}^{i I}(x ; z ; w)(w-x)^{\mu} d z d w, \\
\alpha_{2}^{i}(I) & =-\frac{1}{8} \int W_{10}^{i I \mu}(y ; w)(w-y)_{\mu}(w-y)^{v}(w-y)_{v} d w, \\
\alpha_{3}^{i}(I) & =\frac{1}{8} \int W_{30}^{i I v \mu}{ }_{\mu}\left(y_{1}, y_{2}, y_{3} ; w\right)\left(w-y_{1}\right)_{v} d y_{2} d y_{3} d w .
\end{aligned}
$$

The $L^{0}$ part of (3.5) becomes

$$
\begin{aligned}
& 0=-e \varepsilon(I)+e \sum_{i=3,6} \alpha_{1}^{i}(I), \\
& 0=-2 f(I) \varepsilon(I)+e \sum_{i=3,6} \alpha_{2}^{i}(I), \\
& 0=-4 g(i) \varepsilon(I)+e \sum_{i=3,6} \alpha_{3}^{i}(I) .
\end{aligned}
$$

We shall now show that the limits

$$
\lim _{I \rightarrow-\infty} \alpha_{(\leqq n) J}^{i}(I), \quad i=3,4,5,6 ; \quad J=1,2,3,
$$

exist as a consequence of Lemma 3.1. This being true, (3.12) implies (3.1) for $j=n+1$. 
We have formulas for the kernels $W$,

$$
\begin{aligned}
W_{p q}^{3 I}(\vec{x} ; \vec{y} ; \vec{z} ; w)= & i \int d s V_{p q}^{I}\left(\vec{x} ; \vec{y} ; z_{1}, \ldots, z_{q-1}, s\right) \\
& \times\left[\eta\left(s, z_{q}\right)+M^{I} S_{I}^{h}\left(s, z_{q}\right)\right] \delta\left(z_{q}, w\right)+(\text { sym on } \vec{z}) \\
W_{p q}^{4 I}(\vec{x} ; \vec{y} ; \vec{z} ; w)= & i \int d s \delta\left(w, x_{1}\right)\left[\eta\left(x_{1}, s\right)+M^{I} S_{I}^{h}\left(x_{1}, s\right)\right] \\
& \times V_{p q}^{I}\left(s, x_{2}, \ldots, x_{q} ; \vec{y} ; \vec{z}\right)+(\operatorname{sym} \text { on } \vec{x}) \\
W_{p q}^{5 I}(\vec{x} ; \vec{y} ; \vec{z} ; w)= & -i \int d s d s^{\prime} d s^{\prime \prime} V_{p q+1}^{I}\left(\vec{x}, s ; \vec{y} ; \vec{z}, s^{\prime}\right) \\
& \times\left\{S_{I}\left(s^{\prime}, s^{\prime \prime}\right) \delta\left(s^{\prime \prime}, w\right) \eta\left(s^{\prime \prime}, s\right)-\eta\left(s^{\prime}, s^{\prime \prime}\right) \delta\left(s^{\prime \prime}, w\right) S_{I}^{h}\left(s^{\prime \prime}, s\right)\right. \\
& \left.+M^{I} S_{I}^{s}\left(s^{\prime}, s^{\prime \prime}\right) \delta\left(s^{\prime \prime}, w\right) S_{I}^{h}\left(s^{\prime \prime}, s\right)+M^{I} S_{I}^{h}\left(s^{\prime}, s^{\prime \prime}\right) \delta\left(s^{\prime \prime}, w\right) S_{I}^{s}\left(s^{\prime \prime}, s\right)\right\} \\
& +(\operatorname{sym} \text { on } \vec{x} \text { and } \vec{z})
\end{aligned}
$$

(each $W$ is given by a symmetric sum of which only one term is shown explicitly). The kernel $W^{6}$ involves some substantial Wick ordering, and has the general form

$$
\begin{aligned}
& W_{p q}^{6 I}(\vec{x} ; \vec{y} ; \vec{z} ; w)=-i \sum_{p^{\prime}, p^{\prime \prime}, q^{\prime}, q^{\prime \prime}, i, j, k \geqq 0} \int d \vec{x}^{\prime} d \vec{y}^{\prime} d \vec{z}^{\prime} d \vec{x}^{\prime \prime} d \vec{y}^{\prime \prime} d \vec{z}^{\prime \prime} d s d s^{\prime} d s^{\prime \prime} \\
& \begin{array}{c}
p^{\prime}+p^{\prime \prime}-2 i=p \\
q^{\prime}+q^{\prime \prime}-j-k-1=q
\end{array} \\
& \times\left\{V _ { p ^ { \prime } q ^ { \prime } } ^ { I } \left(x_{1}, \ldots, x_{q^{\prime}-j-1}, x_{1}^{\prime}, \ldots, x_{j}^{\prime}, s ; y_{1}, \ldots, y_{p^{\prime}-i}, y_{1}^{\prime}, \ldots, y_{i}^{\prime} ;\right.\right. \\
& \left.z_{1}, \ldots, z_{q^{\prime}-k}, z_{1}^{\prime}, \ldots, z_{k}^{\prime}\right) \\
& \times V_{p^{\prime \prime} q^{\prime \prime}}^{I}\left(x_{q^{\prime}-j}, \ldots, x_{p}, x_{1}^{\prime \prime}, \ldots, x_{k}^{\prime \prime} ; y_{p^{\prime}-i+1}, \ldots, y_{p}, y_{1}^{\prime \prime}, \ldots, y_{i}^{\prime \prime}\right. \text {; } \\
& \left.z_{q^{\prime}-k+1}, \ldots, z_{q}, z_{1}^{\prime \prime}, \ldots, z_{j}^{\prime \prime}, s^{\prime}\right) \\
& \times\left[\prod_{l=1}^{i} D_{I}^{s}\left(y_{l}^{\prime}, y_{l}^{\prime \prime}\right) \prod_{m=1}^{j} S_{I}^{s}\left(x_{m}^{\prime}, z_{m}^{\prime \prime}\right) \prod_{n=1}^{k} S_{I}^{s}\left(x_{n}^{\prime \prime}, z_{n}^{\prime}\right)\right] \\
& \left.\times\left[S_{I}^{h}\left(s^{\prime}, s^{\prime \prime}\right) \delta\left(s^{\prime \prime}, w\right) \eta\left(s^{\prime \prime}, s\right)-\eta\left(s^{\prime}, s^{\prime \prime}\right) \delta\left(s^{\prime \prime}, w\right) S_{I}^{h}\left(s^{\prime \prime}, s\right)\right]\right\} \\
& +(\operatorname{sym} \text { in } \vec{x}, \vec{y} \text {, and } \vec{z}) \text {. }
\end{aligned}
$$

It is now possible to see the finiteness of the limits (3.13) as a consequence of the integrability properties of the kernels $V_{p q(\leqq n)}^{-\infty}($ Lemma 3.1). For example, consider

$$
\begin{aligned}
\gamma^{\mu} \alpha_{1}^{5}(I)= & -i \int d s d s^{\prime} d z V_{02}^{I}\left(x, s ; z, s^{\prime}\right) \\
& \times\left\{\int d s ^ { \prime \prime } \left[S_{I}\left(s^{\prime}, s^{\prime \prime}\right)\left(s^{\prime \prime}-x\right)^{\mu} \eta\left(s^{\prime \prime}, s\right)-\eta\left(s^{\prime}, s^{\prime \prime}\right)\left(s^{\prime \prime}-x\right)^{\mu} S_{I}\left(s^{\prime \prime}, s\right)\right.\right. \\
& \left.\left.+M^{I} S_{I}^{s}\left(s^{\prime}, s^{\prime \prime}\right)\left(s^{\prime \prime}-x\right)^{\mu} S_{I}^{h}\left(s^{\prime \prime}, s\right)+M^{I} S_{I}^{h}\left(s^{\prime}, s^{\prime \prime}\right)\left(s^{\prime \prime}-x\right)^{\mu} S_{I}^{s}\left(s^{\prime \prime}, s\right)\right]\right\} .
\end{aligned}
$$

A moment's thought tells us that the quantity in braces is smooth and polynomially bounded in the limit $I \rightarrow-\infty$, by the smoothness of the functions $S_{-\infty}^{s}$ and $\eta$ and the exponential decay of $S_{-\infty}^{h}$ and $\eta$. Hence the overall integral is finite up to order $e^{n}$. By the same reason, the limits $\alpha_{(\leqq n) J}^{3}, \alpha_{(\leqq n) J}^{4}, \alpha_{(\leqq n) J}^{5}, J=1,2,3$, all exist.

The $\alpha_{(\leqq n) J}^{6}$ limits are just slightly more difficult. Here we note that a product of any two kernels $V_{p q(\leqq n)}^{-\infty}$ connected by a single line with exponential decay is certainly integrable against smooth polynomially bounded test functions. For each term contributing to $\alpha_{(\leqq n) 1}^{6}(-\infty)$, the connecting line with exponential decay is provided by

$$
\int d s^{\prime \prime}\left[S_{-\infty}^{h}\left(s^{\prime}, s^{\prime \prime}\right)\left(s^{\prime \prime}-x\right)^{\mu} \eta\left(s^{\prime \prime}, s\right)-\eta\left(s^{\prime}, s^{\prime \prime}\right)\left(s^{\prime \prime}-x\right)^{\mu} S_{-\infty}^{h}\left(s^{\prime \prime}, s\right)\right],
$$


and the product of soft propagators is a smooth bounded function of the difference variables, and so the quantity is finite. The limits $\alpha_{(\leqq n) 2}^{6}(-\infty), \alpha_{(\leqq n) 3}^{6}(-\infty)$ follow in the same way.

To complete the proof of Theorem 2.1, we merely note that the Ward identity (2.15) is the $I \rightarrow-\infty$ limit of (2.9).

\section{Proof of Lemma 3.1}

In the following argument, we have implicitly applied the gauge invariant loop/photon regularization used in [FHRW, Sects. 3-5]. Then $V_{\infty}^{I}$ is finite and has gauge invariant form

$$
V_{\infty}^{I}(\Phi)=-e \tilde{c}(I) V_{5}-\tilde{b}(I) V_{1}-\tilde{a}(I) V_{4}-\tilde{d}(I) V_{3},
$$

and satisfies the " $Z_{1}=Z_{2}$ " condition:

$$
\tilde{c}(I)^{-1}(1+\tilde{a}(I))=c(I)^{-1}(1+a(I))=\varepsilon(I) .
$$

We make a change of variables $\psi(x)=e^{i e x(x)} \psi^{\prime}(x), \tilde{\psi}(x)=\tilde{\psi}^{\prime}(x) e^{-i e x(x)}, A=A^{\prime}$ in the functional integral

$$
V_{0}^{I}\left(\Phi^{e}\right)=\log \int d P^{h}(\Phi) \exp V_{\infty}^{I}\left(\Phi+\Phi^{e}\right) .
$$

We calculate

$$
\begin{aligned}
V_{\infty}^{I}\left(A+A^{e}, \tilde{\psi}+\tilde{\psi}^{e}, \psi+\psi^{e}\right)= & V_{\infty}^{I}\left(A^{\prime}+A^{e}, \tilde{\psi}^{\prime}+\tilde{\psi}^{e} e^{i e \chi}, \psi^{\prime}+e^{-i e \chi_{\psi} e}\right) \\
& -e \tilde{a}(I) V_{5}\left(\partial \chi, \tilde{\psi}^{\prime}+\tilde{\psi}^{e} e^{i e \chi}, \psi^{\prime}+e^{-i e \chi_{\psi} e}\right),
\end{aligned}
$$

and

$$
\begin{aligned}
d P^{h}(\Phi)= & d P^{h}\left(\Phi^{\prime}\right) \exp -\left\{\int \tilde{\psi}^{\prime}\left[e^{-i e \chi} R_{I} e^{i e \chi}-R_{I}\right] \psi^{\prime}+\int \tilde{\psi}^{\prime} e \phi \chi \psi^{\prime}\right\} \\
= & d P^{h}\left(\Phi^{\prime}\right) \exp -\left\{\int \tilde{\psi}^{\prime}\left[e^{-i e \chi} R_{I} e^{i e \chi}-R_{I}\right] \psi\right. \\
& \left.+e V_{5}\left(\partial \chi, \tilde{\psi}^{\prime}+\tilde{\psi}^{e} e^{i e \chi}, \psi^{\prime}+e^{-i e \chi} \psi^{e}\right)\right\} \\
& \times \exp e \int\left[\tilde{\psi}^{\prime} \phi \chi e^{-i e \chi} \psi^{e}+\tilde{\psi}^{e} e^{i e \chi} \phi \chi \psi^{\prime}+\tilde{\psi}^{e} e^{i e \chi} \phi \chi e^{-i e \chi} \psi^{e}\right],
\end{aligned}
$$

where $R_{I}(x, y) \equiv\left[S_{I}^{h^{-1}}-S_{I}^{-1}\right](x, y)$. Since the $V_{3}$ term in (4.3) is gauge-invariant, the $V_{5}$ terms can be combined with $V_{\infty}^{I}$, and the change of variables yields the identity

$$
\begin{aligned}
V_{0}^{I}\left(\Phi^{e}\right)= & \log \int d P^{h}\left(\Phi^{\prime}\right)\left\{\exp -\int \tilde{\psi}^{\prime}\left[e^{-i e \chi} R_{I} e^{i e \chi}-R_{I}\right] \psi^{\prime}\right. \\
& \times \exp e \int\left[\tilde{\psi}^{\prime} \phi \chi e^{-i e \chi} \psi^{e}+\tilde{\psi}^{e} e^{i e \chi} \phi \chi \psi^{\prime}+\tilde{\psi}^{e} e^{i e \chi} \phi \chi e^{-i e \chi} \psi^{e}\right] \\
& \left.\times \exp V_{\infty}^{I}\left(A^{\prime}+A^{e}+\tilde{c}(I)^{-1}(1+\tilde{a}(I)) \partial \chi, \tilde{\psi}^{\prime}+\tilde{\psi}^{e} e^{i e \chi}, \psi^{\prime}+e^{-i e \chi} \psi^{e}\right)\right\} .
\end{aligned}
$$

The key feature of this formula is the appearance of $\tilde{c}, \tilde{a}$ only in the combination $\tilde{c}^{-1}(1+\tilde{a})=\varepsilon$. The term linear in $\chi$ is extracted by application of the operator $\int \chi(x)\left[\left.\frac{\delta}{\delta \chi(x)} \cdot\right|_{\chi=0}\right] d x$ :

$$
\begin{aligned}
0= & {\left[\exp V_{0}^{I}\left(\Phi^{e}\right)\right]^{-1}\left[\int d P^{h}\left(\Phi^{\prime}\right) \exp V_{\infty}^{I}\left(\Phi^{\prime}+\Phi^{e}\right)\right.} \\
& \times\left\{i e \int \tilde{\psi}^{\prime}\left[\chi R_{I}-R_{I} \chi\right] \psi^{\prime}+e \int\left[\tilde{\psi}^{\prime} \phi \chi \psi^{e}+\tilde{\psi}^{e} \phi \chi \psi^{\prime}+\tilde{\psi}^{e} \phi \chi \psi^{e}\right]\right\} \\
& +\left.\int \chi\left[\frac{\delta}{\delta \chi} V_{0}^{I}\left(A^{e}+\varepsilon(I) \partial \chi, \tilde{\psi}^{e} e^{i e \chi}, e^{-i e \chi} \psi^{e}\right)\right]\right|_{\chi=0} .
\end{aligned}
$$


With the notation introduced in Lemma 3.2, and the integration by parts formulas,

$$
\begin{gathered}
{\left[\exp V_{0}^{I}\left(\Phi^{e}\right)\right]^{-1} \int d P^{h}(\Phi) \tilde{\psi}(y) \exp V_{\infty}^{I}\left(\Phi+\Phi^{e}\right)} \\
\quad=\int\left[\partial_{\psi} V\right]\left(\Phi^{e}, x\right) S_{I}^{h}(x, y) d x, \\
{\left[\exp V_{0}^{I}\left(\Phi^{e}\right)^{-1} \int d P^{h}(\Phi) \psi(x) \exp V_{\infty}^{I}\left(\Phi+\Phi^{e}\right)\right.} \\
\quad=\int S_{I}^{h}(x, y)\left[\partial_{\tilde{\psi}} V\right]\left(\Phi^{e}, y\right) d y, \\
{\left[\exp V_{0}^{I}\left(\Phi^{e}\right)\right]^{-1} \int d P^{h}(\Phi) \psi(x) \tilde{\psi}(y) \exp V_{\infty}^{I}\left(\Phi+\Phi^{e}\right)} \\
=S_{I}^{h}(x, y)+\int S_{I}^{h}\left(x, x^{\prime}\right)\left\{\left[\partial_{\tilde{\psi} \psi}^{2} V\right]\left(\Phi^{e}, x^{\prime}, y^{\prime}\right)\right. \\
\left.\quad+\left[\partial_{\tilde{\psi}} V\right]\left(\Phi^{e}, x^{\prime}\right)\left[\partial_{\psi} V\right]\left(\Phi^{e}, y^{\prime}\right)\right\} S_{I}^{h}\left(y^{\prime}, y\right) d x^{\prime} d y^{\prime},
\end{gathered}
$$

Eq. (4.5) becomes

$$
\begin{aligned}
& -i e \int \operatorname{Tr}\left[S_{I}^{h}(x, y)+\int S_{I}^{h}(x, z)\right. \\
& \left.\quad \times\left\{\left[\partial_{\tilde{\psi} \psi}^{2} V\right]\left(\Phi^{e}, z, w\right)+\left[\partial_{\tilde{\psi}} V\right]\left(\Phi^{e}, z\right)\left[\partial_{\psi} V\right]\left(\Phi^{e}, w\right)\right\} S_{I}^{h}(w, y) d z d w\right] \\
& \quad \times\left[\chi(y) R_{I}(y, x)-R_{I}(y, x) \chi(x)\right] d x d y \\
& \quad+e \int\left[\partial_{\psi} V\right]\left(\Phi^{e}, x\right)\left[S_{I}^{h}(x, y) \phi \chi(y)-i \delta(x, y) \chi(y)\right] \psi^{e}(y) d x d y \\
& \quad+e \int \tilde{\psi}^{e}(x)\left[\phi \chi(x) S_{I}^{h}(x, y)+i \chi(x) \delta(x, y)\right]\left[\partial_{\tilde{\psi}} V\right]\left(\Phi^{e}, y\right) d x d y \\
& \quad+e \int \tilde{\psi}^{e}(x) \phi \chi(x) \psi^{e}(x) d x \\
& \quad+\varepsilon(I) \int\left[\partial_{A} V\right]\left(\Phi^{e}, x\right) \partial \chi(x) d x=0 .
\end{aligned}
$$

Next, we note the convolution formula

$$
R_{I} * S_{I}^{h}=S_{I}^{h} * R_{I}=\eta \mathbf{1}
$$

and the identities

$$
\begin{aligned}
& e \int\left[\phi \chi(x) S_{I}^{h}(x, y)+i \chi(x) \delta(x, y)\right] d x \\
& \quad=i e \int \chi(x)\left[\eta(x, y)+M^{I} S_{I}^{h}(x, y)\right] d x, \\
& e \int\left[S_{I}^{h}(x, y) \phi \chi(y)-i \delta(x, y) \chi(y)\right] d y \\
& \quad=i e \int\left[\eta(x, y)+M^{I} S_{I}^{h}(x, y)\right] \chi(y) d y .
\end{aligned}
$$

Their insertion into (4.7) yields

$$
\begin{aligned}
0= & -i e(\operatorname{Tr} 1) \int[\chi(y)-\chi(x)] \eta(y, x) d x d y \\
& -i e \int \operatorname{Tr}\left\{\left[\partial_{\tilde{\psi} \psi}^{2} V\right]\left(\Phi^{e}, x, y\right) \chi(z)\right. \\
& \left.\times\left[S_{I}^{h}(y, z) \eta(z, x)-\eta(y, z) S_{I}^{h}(z, x)\right]\right\} d x d y d z \\
& -i e \int \chi(z)\left[\partial_{\psi} V\right]\left(\Phi^{e}, y\right) \\
& \times\left[S_{I}^{h}(y, z) \eta(z, x)-\eta(y, z) S_{I}^{h}(z, x)\right]\left[\partial_{\tilde{\psi}} V\right]\left(\Phi^{e}, x\right) d x d y d z \\
& +i e \int \tilde{\psi}^{e}(x) \chi(x)\left[\eta(x, y)+M^{I} S_{I}^{h}(x, y)\right]\left[\partial_{\tilde{\psi}} V\right]\left(\Phi^{e}, y\right) d x d y \\
& +i e \int\left[\delta_{\psi} V\right]\left(\varphi^{e}, x\right)\left[\eta(x, y)+M^{I} S_{I}^{h}(x, y)\right] \chi(y) \psi^{e}(y) d x d y \\
& +e \int \tilde{\psi}^{e}(x) \oplus \chi(x) \psi^{e}(x) d x \\
& +\varepsilon(I) \int\left[\partial_{A} V\right]\left(\Phi^{e}, x\right) \partial \chi(x) d x .
\end{aligned}
$$


The first term vanishes and the fourth and fifth terms can be Wick ordered using the identities:

$$
\begin{aligned}
& \tilde{\psi}^{e}(x)\left[\partial_{\tilde{\psi}} V\right](y)=: \tilde{\psi}^{e}(x)\left[\partial_{\tilde{\psi}} V\right](y):-\int\left[\partial_{\tilde{\psi} \psi}^{2} V\right](y, z) S_{I}^{s}(z, x) d z, \\
& {\left[\partial_{\psi} V\right](x) \psi^{e}(y)=:\left[\partial_{\psi} V\right](x) \psi^{e}(y):-\int S_{I}^{s}(y, z)\left[\partial_{\psi \tilde{\psi}}^{2} V\right](z, x) d z,}
\end{aligned}
$$

and so formula (3.5) is obtained.

Acknowledgements. I am grateful for many discussions with my colleagues, especially with Prof. Lon Rosen and Prof. Joel Feldman, who were unstinting in their helpful suggestions and criticisms during the preparation of this manuscript.

\section{References}

[BM] Breitenlohner, P., Maison, D.: Dimensional renormalization and the action principle. Commun. Math. Phys. 52, 11-38 (1977)

Breitenlohner, P., Maison, D.: Dimensionally renormalized Green's functions for theories with massless particles. I and II. Commun. Math. Phys. 52, 39-54 and 55-75 (1977)

[BRS] Becchi, C., Rouet, A., Stora, R.: Renormalizable models with broken symmetries. In: Renormalization theory. (Erice 1975), Velo, G., Wightman, A.S. (eds.). Dordrecht: Reidel 1976

[FHRW] Feldman, J., Hurd, T., Rosen, L., Wright, J.: QED: a proof of renormalizability. Lecture Notes in Physics, vol. 312. Berlin, Heidelberg, New York: Springer 1988

[GN] Gallavotti, G., Nicolo, F.: Renormalization theory in four-dimensional scalar fields. I and II. Commun. Math. Phys. 100, 545-590 (1985) and 101, 247-282 (1986)

['tHV] 't Hooft, G., Veltman, M.: Regularization and renormalization of gauge fields. Nucl. Phys. B 44, 189-213 (1972)

Communicated by K. Gawedzki

Received June 6, 1988 
